\title{
Percepciones sobre la alimentación saludable y sus implicaciones en la
cocina doméstica en un grupo de mujeres de Cataluña, España \\ Percepciones sobre la alimentación saludable y sus implicaciones en la
cocina doméstica en un grupo de mujeres de Cataluña, España
}

DOI: 10.17533/udea.penh.v22n1a02

PERSPECTIVAS EN NUTRICIÓN HUMANA

ISSN 0124-4108

Escuela de Nutrición y Dietética, Universidad de Antioquia. Medellín, Colombia

Vol. 22, N. ${ }^{\circ}$, enero-junio de 2020, pp. 19-34.

Artículo recibido: 10 de febrero de 2020

Aprobado: 5 de junio de 2020

\section{Leyna Priscila López Torres ${ }^{1 * ;}$, Jesús Contreras Hernández²}

\section{- - Resumen}

Antecedentes: por lo general, y entre otras consideraciones, las personas declaran tomar en cuenta el valor de la salud cuando eligen sus alimentos. Objetivo: comprender, desde la perspectiva de un grupo de mujeres, lo que constituye una alimentación saludable y sus implicaciones en las prácticas culinarias cotidianas. Materiales y métodos: con orientación fenomenológica y metodologías cualitativas, guiadas por el criterio de saturación de la información, se realizaron cuatro grupos de discusión ( $n=22)$ y 10 entrevistas en profundidad $(n=12)$ a 31 mujeres residentes en Cataluña, España. Resultados: las percepciones sobre "lo saludable" son diversas y complejas. Además de identificarse la reproducción de discursos que denotan la nutricionalización de la alimentación, también se encontraron procesos de asimilación y resignificación que influencian la percepción de "lo saludable" y las prácticas culinarias. Antes que la salud, se consideran las preferencias, los gustos, el costo y la conveniencia en las elecciones alimentarias. Conclusión: las resignificaciones sobre lo que es considerado como "saludable" permite a las entrevistadas integrar estrategias mediante las cuales legitiman los discursos sobre salud en sus prácticas culinarias, aunque dichas estrategias no necesariamente se correspondan con las normas dietéticas.

Palabras clave: alimentación saludable, dieta, percepción, salud, cocina doméstica, consumos alimentarios.

$1^{*}$ Autor de correspondencia. Profesora docente asociada "C" del Departamento de Ciencias Sociales, Centro Universitario de Ciencias de la Salud, Universidad de Guadalajara. Doctora en Alimentación y Nutrición, Universitat de Barcelona, en Barcelona, España. Orcid: https://orcid.org/0000-0002-5644-8416. Correo electrónico: priscila.lopez@academicos.udg.mx

2 Profesor emérito del Departamento de Antropología Social, Facultad de Geografía, Universidad de Barcelona. Doctorado en Filosofía y Letras Universitat de Barcelona, en Barcelona, España.

Cómo citar este artículo: López Torres LP, Contreras Hernández J. Percepciones sobre la alimentación saludable y sus implicaciones en la cocina doméstica en un grupo de mujeres de Cataluña, España. Perspect Nutr Humana. 2020; 22:19-34. DOI: 10.17533/udea.penh.v22n1a02 
Percepciones sobre la alimentación saludable

\section{Perceptions Around Healthy Eating and Implications for Home Cooking in a Group of Women in Catalonia, Spain}

\section{Abstract}

Background: In general, and among various factors, most people declare that they take into account the importance of health when they are choosing their foods. Objective: Understand from the perspective of a group of women what constitutes healthy eating and the implications for daily cooking practices. Objective: Understand from the perspective of a group of women what constitutes healthy eating and the implications for daily cooking practices. Materials and Methods: With phenomenological orientation and qualitative methodologies, guided by the information saturation criterion, four discussion groups $(n=22)$ and 10 in-depth interviews $(n=12)$ were conducted with 31 women residing in Catalonia, Spain. Results: Perceptions around what is "healthy" are diverse and complicated. In addition to identifying how discourse denotes whether foods are healthy or not, it was also found that assimilation and redefinition processes influence perceptions of "healthy" foods and culinary practices. Before considering health, preferences, tastes, cost, and convenience were considered when selecting food and making purchases. Conclusion: Helping the participants to identify how they label and consider "healthy" foods enabled the integration by interviewees of strategies to legitimize their discussions about health and culinary practices, although said strategies do not necessarily correspond with dietetic norms.

Keywords: Healthy eating, diet, perception, health, home cooking, food consumption.

\section{INTRODUCCIÓN}

La vinculación entre alimentación y salud existe desde el principio de las civilizaciones humanas, pero, en el marco de la modernidad alimentaria, ${ }^{1}$ los discursos sobre alimentación se han medicalizado (1): los alimentos son aprehendidos, fundamentalmente, como agregados de nutrientes bioquímicos que es necesario equilibrar para vivir con buena salud e insistiendo en el impacto positivo de ciertos componentes de los alimentos sobre la salud de las personas y su bienestar en general.

En este contexto, la racionalidad nutricional privilegia la relación entre el valor nutricional y la salud como la condición suprema en la alimentación (2),

1 Concepto bajo el cual se denomina el periodo alimentario actual, caracterizado por las variaciones en las maneras de alimentarse de las personas, debido a los cambios sociales y demográficos experimentados en diferentes sociedades, a partir de los procesos de industrialización, urbanización, avances del ámbito tecnológico y médico, entre otros. ello se aprecia en los discursos de los españoles, quienes declaran que la salud es un factor relevante al momento de elegir sus alimentos (3-5), y las mujeres, por diferentes razones, le conceden mayor importancia que los hombres (5-7).

Por otro lado, y como es sabido, existen diferencias entre los discursos y las prácticas alimentarias de las personas (8,9), y una desviación entre los consumos alimentarios declarados y la calidad nutricional de estos $(3,10,11)$. Al respecto, Varela-Moreiras et al. (12) identificaron que las dietas de los españoles superaban en cantidad las pautas nutricionales recomendadas de consumo de carne y sus derivados, por oposición a ingestas insuficientes de cereales y derivados, verduras, frutas y legumbres.

En el hogar se consume el $80 \%$ de los alimentos, transformados de manera culinaria (10). Cocinar es una actividad predominantemente femenina $(8,13)$ 
y son ellas, las mujeres, quienes conceden mayor importancia a la salud en la alimentación que los hombres. Por estas razones, el presente estudio tuvo como objetivo comprender, desde la perspectiva de un grupo de mujeres, lo que constituye una alimentación "saludable" y sus implicaciones en las prácticas culinarias cotidianas (PCC), entendidas como las actividades relacionadas con la planificación, adquisición (de suministros) y elaboración de las comidas en el hogar.

\section{MATERIALES Y MÉTODOS}

La investigación se realizó con perspectiva fenomenológica, que estudia "las realidades cuya naturaleza y estructura son construidas por el sujeto que las vive y las experimenta" (14, p. 137) a través de la comunicación e interacción con los demás. Para ello, con un diseño cualitativo se realizaron grupos de discusión (GD) y entrevistas en profundidad (EP).

A partir de los trabajos de Contreras et al. (3), Luce Giard (15) y Frances Short (16), se diseñó un guion de preguntas para el desarrollo de los GD y las EP. Dicho guion solo sirvió de guía, ya que tanto los GD como las EP se desarrollaron con la suficiente flexibilidad para permitir que emergieran cuestiones no previstas que ayudaran a la comprensión del fenómeno de estudio.

Los GD permitieron explorar ${ }^{3}$ la temática, mientras que en las EP se indagó con más detalle en los temas que fueron surgiendo durante el trabajo de campo.

2 El término saludable se presenta entre comillas dado que es una construcción que responde a distintas apreciaciones y no solo desde la óptica de la nutrición.

3 La flexibilidad con la que se condujeron los GD favoreció las interacciones entre las participantes (se cuestionaron entre sí, compartieron y contrastaron sus opiniones y experiencias), dada la heterogeneidad de los perfiles de las informantes; ambas condiciones permitieron que emergieran las temáticas de interés.
La conformación de los GD y las EP fue a partir de distintas formas de reclutamiento: a) entrega de invitaciones impresas y carteles pegados en distintos centros cívicos de Barcelona; b) convocatoria en la aplicación WiNK; y c) por el método "bola de nieve" (17). Los criterios de inclusión fueron mujeres entre 23 a 73 años edad, residentes en Cataluña y que reconocieran cocinar en casa, al menos en una toma alimentaria al día.

Los GD y las EP se audiograbaron y transcribieron en su totalidad. El tiempo promedio de duración de los GD y las EP fue de 2:14 h y 1:30 h, respectivamente.

El análisis de los datos fue realizado en paralelo a la conducción del trabajo de campo, según el método de comparación constante (18). La saturación de la información (17) fue el parámetro empleado para finalizar la recolección de datos.

Se realizaron $4 \mathrm{GD}(\mathrm{n}=22)$ y $10 \mathrm{EP}(\mathrm{n}=12)^{4}$ de septiembre del 2011 a octubre del 2014. En total participaron 31 mujeres $^{5}$ residentes de cinco municipios (Barcelona, Masnou, Badalona, San Cugat del Vallès y Cerdenyola) de Cataluña, España.

Tres GD se realizaron en espacios reservados para este fin y un cuarto, en un bar. Las EP se condujeron en los centros de labores de las participantes o bares cercanos a los domicilios 0 a los centros de trabajo de las informantes. El modelo de decisiones alimentarias de Sobal et al. (19) fue el referente teórico que orientó el proceso de análisis. ${ }^{6}$ Tres conceptos constituyeron la guía fundamental para la aproximación a los datos.

4 Dos de las 10 EP se realizaron en presencia de madre e hija, el resto fueron individuales.

5 Tres de las mujeres participaron tanto en los GD como en las $E P$, por considerarlas informantes clave.

6 Su elección se debió a la perspectiva histórica, social y cultural que se operacionaliza en el modelo bajo una óptica de itinerario vital; condiciones que permiten la exploración de la complejidad en las elecciones alimentarias. 
El primero, el sistema personal de valores, se refiere a las consideraciones, significados y sentimientos que las personas experimentan al realizar sus elecciones alimentarias, se ubica aquí a la salud. El segundo corresponde a las transiciones o sucesos que provocan inflexiones en la vida de las personas y repercuten en el tercer concepto: en las trayectorias alimentarias, es decir, en las maneras de pensar, sentir y actuar de las personas en torno a su alimentación.

A partir del método de análisis empleado (18), la codificación de los datos (reducción analítica de la información) implicó la detección y comparación de las diferencias y las semejanzas de lo percibido "saludable" y su influjo en las PCC. Así, se construyeron categorías (códigos relacionados) y familias (categorías relacionadas) relativos a las temáticas estudiadas.

Como criterios de validez interna del proceso de análisis se plantearon dos estrategias: la primera fue la socialización del proceso de recolección y análisis de los datos con otros investigadores ${ }^{7}$ y la segunda, la retroalimentación de las informantes durante los GD y las EP. ${ }^{8}$

La gestión y análisis de los datos se realizó en el programa ATLAS-Ti v.6.

En la tabla 1, se presentan los datos que permiten la caracterización de las participantes. Para preservar las identidades de las mujeres les fue asignado un seudónimo.

$7 \quad$ El segundo autor del artículo y otros miembros del Observatorio de la Alimentación de la Universitat de Barcelona.

8 Las segundas preguntas permitieron confirmar o hacer precisiones 0 aclaraciones sobre las ideas expresadas por las informantes, por ejemplo, de acuerdo a lo que comentas, entiendo que tú consideras que ¿una pizza hecha en casa es más saludable que una pizza que compras hecha? Entonces, en tu caso, ¿percibes que es más saludable un alimento que se produce en la región en contraste con uno que no? ¿Estoy en lo correcto?

\section{Consideraciones éticas}

El trabajo fue aprobado por la Dirección del Observatorio de la Alimentación de la Universitat de Barcelona, guiado por los principios éticos internacionales (20) y las recomendaciones para estudios con perspectiva social (21). Las mujeres fueron informadas sobre el objetivo de la investigación y todas firmaron su anuencia de participación en un consentimiento informado.

\section{RESULTADOS}

En las PCC, como manifestación de las decisiones alimentarias de las informantes, se detectó que la salud es un valor entre otros que conforman el sistema personal de decisiones alimentarias (SPDA).

Para entender cómo la salud influye en las PCC, en primer lugar, se presenta cómo el valor de la salud es percibido y resignificado y, a continuación, se presentan las estrategias que las informantes señalaron llevar a cabo en su cotidianidad cuando consideran la salud en sus decisiones. Finalmente, se expone la complejidad de la relación entre la salud y el resto de valores que conforman el SPDA para entender algunas de las razones de los desfases entre lo que se piensa y lo que se hace en torno a la salud y las decisiones alimentarias.

\section{Percepciones sobre lo que constituye una ali- mentación saludable}

La construcción de la salud, como valor del SPDA, se realizó a partir de lo que es percibido por las participantes como "sano", "saludable", "nutritivo", "que nutre" o aquello que se considera tiene un impacto en la salud, lo "bueno" o "malo" para el cuerpo o su integridad física. Así, se identificaron tres ejes argumentales. 
Tabla 1. Perfiles de las informantes que conformaron la muestra estudiada

\begin{tabular}{|c|c|c|c|c|c|c|}
\hline Pseudónimo & País de nacimiento & Edad & Situación laboral* & Nivel de estudio† & Conformación del hogarł & Formó parte de $\S$ \\
\hline Esther & Perú & 73 & $\mathrm{H}$ & GS & $P$ & GD \\
\hline Dulce & España & 69 & $\mathrm{H}$ & $\mathrm{P}$ & $S$ & EP \\
\hline María & España & 64 & $\mathrm{H}$ & NC & $P$ & GD \\
\hline Sara & Chile & 57 & $\mathrm{E}$ & $\mathrm{P}$ & $\mathrm{PH}$ & EP \\
\hline Cristina & España & 54 & $\mathrm{JC}$ & $S$ & $P$ & GD \\
\hline Pilar & España & 51 & JC & $\mathrm{MD}$ & $S$ & EP \\
\hline Sonia & España & 50 & $\mathrm{JC}$ & $S$ & $P$ & GD \\
\hline Antonia & España & 48 & JC & GS & $\mathrm{PH}$ & EP \\
\hline Isabel & España & 48 & E & GS & $\mathrm{PH}$ & EP \\
\hline Manuela & Francia & 44 & MJ & $S$ & $\mathrm{PH}$ & GD \\
\hline Mercedes & Argentina & 45 & $\mathrm{P}$ & NC & $S$ & GD \\
\hline Alba & Portugal & 44 & MJ & $\mathrm{MD}$ & S & GD \\
\hline Mónica & España & 44 & JC & GS & $\mathrm{PH}$ & GD \\
\hline Eva & España & 43 & $\mathrm{JC}$ & $S$ & $\mathrm{PH}$ & GD y EP \\
\hline Alicia & Italia & 43 & $E$ & U3 & $\mathrm{CP}$ & $\mathrm{GD}$ \\
\hline Yolanda & España & 42 & $\mathrm{JC}$ & $S$ & $\mathrm{MH}$ & GD y EP \\
\hline Beatriz & España & 42 & $\mathrm{H}$ & GS & $\mathrm{CP}$ & GD \\
\hline Milagros & España & 42 & E & MD & PA & EP \\
\hline Noelia & Francia & 42 & MJ & GS & $P$ & GD \\
\hline Verónica & España & 40 & $\mathrm{JC}$ & $S$ & $S$ & GD \\
\hline Carmen & España & 39 & MJ & U3 & $\mathrm{CP}$ & GD \\
\hline Josefa & Colombia & 33 & MJ & $\mathrm{MD}$ & $S$ & GD \\
\hline Nuria & España & 31 & $\mathrm{JC}$ & $\mathrm{MD}$ & $S$ & GD \\
\hline Laura & España & 30 & $E$ & GS & $P$ & GD \\
\hline Raquel & España & 30 & $\mathrm{H}$ & $S$ & $\mathrm{CP}$ & GD \\
\hline Lucía & Colombia & 29 & JC & $\mathrm{MD}$ & $\mathrm{CP}$ & GD \\
\hline Irene & Colombia & 29 & MJ & $M D$ & $\mathrm{CP}$ & GD \\
\hline Claudia & Argentina & 28 & JC & $\mathrm{MD}$ & $P$ & EP \\
\hline Rosario & Estados Unidos & 27 & MJ & U3 & $\mathrm{CP}$ & GD \\
\hline Juana & España & 25 & MJ & U3 & $\mathrm{CP}$ & EP \\
\hline Julia & España & 24 & $\mathrm{JC}$ & $M D$ & $S$ & EP \\
\hline
\end{tabular}


Alimentos, nutrientes, frecuencias y cantidades de consumo. Si bien alimentos como judías (también conocidas como frijoles), garbanzos, lentejas, carne de cerdo, de res, de pollo, pescado blanco o azul, frutas, verduras y hortalizas se consideraron "saludables"; como "no saludables", nata, bollería, galletas y aceites vegetales (con excepción del aceite de oliva); y algunos, como la leche, como alimentos ambivalentes, algunas mujeres percibieron este último como un alimento "saludable", otras relacionaron su consumo (necesario) solo durante la infancia y no así durante la adultez, ya fuera por considerarla de difícil digestión o por relacionarla con métodos masivos de producción de alimentos, como se verá más adelante.

En cuanto a nutrientes específicos, aunque se mencionó la importancia del consumo de carbohidratos, fibra e incluso de vitaminas (sin particularizar ninguna), se coincidió en identificar tanto la carencia de grasa como el empleo mínimo de esta como una condición asociada con una alimentación saludable en alimentos, técnicas culinarias, platos, etc.

Sobre la grasa, algunas informantes diferenciaron las grasas saturadas o "malas" y las "buenas" o "beneficiosas". La grasa saturada es percibida como mala para la salud por asociarse con efectos adversos en el cuerpo. Los más señalados son el aumento de peso y la obesidad.

Sobre las frecuencias de consumos, las ingestas alimentarias se circunscriben en términos de periodos de tiempo, generalmente semanal, y cantidades de consumo para alimentos y modos de preparación.

yo pienso en la semana, no en el día, en la semana, yo hago un repaso de la semana y digo: "esta semana mis hijos han comido legumbres, han comido arroz, han comido verduras, han comido frutas, han comido pescados" y tiene que haber de todo a lo largo de la semana [lsabel, 48 años].

El tipo y combinación de alimentos, las maneras en cómo son convertidos en platos y las frecuencias y cantidades en que estos son ingeridos se asociaron con patrones de consumo, para los que las ideas de equilibrio y variedad fueron las constantes en los discursos.

La variedad se expresa en tres dimensiones: 1) incluir alimentos de distintos grupos (frutas, verduras, legumbres); 2) consumir diferentes alimentos de un mismo grupo; e 3) incluir diversas formas de preparación de un mismo alimento (por ejemplo, arroz con verduras, arroz con setas 0 arroz con tomate).

En cuanto a "tener una alimentación equilibrada", se consideraron las cantidades y las frecuencias de consumo, para variar la alimentación. Se identificaron dos funciones de la variedad: la posibilidad de incluir distintos nutrientes y diversificar los consumos alimentarios para evitar el "aburrimiento de comer siempre lo mismo".

Aproximadamente la mitad de las informantes señaló el modelo de la "dieta mediterránea" como ejemplo de patrón alimentario saludable, pues las mujeres perciben que incluye tipos de alimentos, frecuencias y modos de preparar los alimentos, considerados, todos ellos, como "saludables".

Que haya un poco de todo, que haya proteínas, es decir, que haya carne, pescado, todo... verduras, lácteos... Que se coma variado, de todo. Lo que tenemos muy bien aquí es que la dieta mediterránea es muy rica [...] [hay] mucha verdura, mucha cosa cruda, por decirlo de alguna manera, lo tienes todo, pescado, carne, hay mucha variedad, mucha fruta [Eva, 43 años].

Al planificar las comidas, las informantes revelaron su intencionalidad de variar y equilibrar la 
alimentación. La planificación involucra procesos mentales mediante los cuales las participantes concilian diversos factores para decidir qué insumos adquirir o qué platos elaborar.

Aunque la variedad fue asociada como característica de la alimentación saludable y la planificación el medio para conseguirla, pensar la comida, es decir, qué platos hacer, también fue percibida como una de las actividades más pesada, debido a la necesidad de tener en cuenta diferentes consideraciones al proyectar los platos que serán elaborados: satisfacción de los gustos y preferencias propias y de otros; cumplir con los ideales propios y de otros, relativos a cada toma alimentaria; la gestión de recursos como tiempo y dinero, entre otras.

Aunque importante discursivamente, al final, la variedad es una condición más aspiracional que real, resignificada en las PCC de las entrevistadas. Por ejemplo, algunas mujeres destacaron renunciar al control de la planificación y acudir a otros por ideas; otras señalaron implementar una variedad "relativa", que se repite (selección de alimentos y platos) cada semana, o simplemente consumir de alimentos o platos en días consecutivos.

[Piensas] ¿Qué haces? De primero y de segundo, pero claro [...] es complicado. No es tan, tan fácil. Cada día, cada día de tu vida planificar el primero $y$ el segundo [...]. Yo no soy de planificar, yo no diría el lunes voy a comer lentejas, el martes... yo digo ¿qué cómo? y a lo mejor en toda la semana he comido tres días lo mismo y es lo que como yo [Yolanda, 42 años].

[...] me canso de pensar en qué hacer y hacer la comida, o sea, hacerla es fácil, lo complicado es pensar en que tienes que hacer y tener de todo en la cocina para poderlo hacer cuando llegas ino? Para mí esto es lo peor [...] repito mucho [...] cada semana habrá lo mismo, ternera y patatas [...], pasta,
[...] crema de verduras y [...] nuggets y patatas fritas [Antonia, 48 años].

Cómo, con qué y en dónde se elaboran los alimentos. En términos generales, las técnicas culinarias o modos de elaboración de los alimentos que integran una alimentación saludable fueron crudo, salteado, al vapor, hervido, al horno, a la plancha y los guisos, ${ }^{9}$ por oposición a los fritos, las salsas ${ }^{10} 0$ hervir "demasiado" los vegetales. La principal razón para catalogar las técnicas de preparación como "saludables" se relacionó con el contenido de materia grasa empleado; cuanto menor sea, la preparación se percibe como más "saludable".

¿Más saludables? a ver, preparaciones de alimentos [...] en cuanto a verduras yo creo que crudo sería lo ideal, bueno las que se puedan comer crudas, porque conservan más vitaminas; después, diría al vapor, porque conserva más que hervir, el horno porque le ahorras toda la materia grasa y el salteado también se puede hacer con poco aceite, creo que son las que requieren menos cantidad de materia grasa ¿no? Las más saludables y las que preservan más la característica del alimento que no se pierde todo o en el agua o en el aceite [Claudia,28 años].

Sobre el equipo y menaje de cocina empleado para la elaboración de las comidas se presentaron tensiones y contradicciones que influyen en la percepción de las mujeres. Los elementos más controvertidos fueron el uso de "la silicona"11 y del horno microondas. La mayoría de las informantes asoció su uso con la facilidad para hacer

9 Platos en los que se combinan productos cárnicos y alimentos vegetales (legumbres, verduras, etc.).

10 Hechas a base de lácteos (nata, mantequilla), oleaginosas (nueces, almendras, etc.) 0 aceite en exceso. Sofritos y salsas blancas como la bechamel fueron las más mencionadas.

11 También fue referenciada con el nombre de una casa comercializadora de dicho producto. Se refiere a un estuche (en forma de contenedor) de una sola pieza, flexible, hecho de silicona, tiene dos hojas superiores que se solapan y con su movimiento es posible abrirlo y cerrarlo. 
preparaciones saludables (poco o nulo empleo de grasa). Pero, para algunas, el uso de la silicona y del microondas levantaba sospechas sobre su inocuidad, por considerar la posible transferencia de sustancias al emplear la silicona para cocinar los alimentos o la apreciación de que, con los microondas, los alimentos sufren cambios en su composición y esto podría tener efectos negativos en la salud.

la silicona no me acaba de convencer, el microondas no me ha convencido [...] Porque no se sabe lo que traspasa, yo soy fumadora, [...] yo tengo mis vicios [y] no voy a añadir más toxinas. Estas que tengo y ya está [risas] ya tengo mi dosis de toxinas al día [Noelia, 42 años].

Con relación al lugar de elaboración de los platos, la comida "hecha en casa" se aprecia como más "natural" y "saludable" frente a la comida elaborada fuera de esta (bares, restaurantes, empresas). Las informantes sostienen que, cuando ellas elaboran sus platos, conocen y controlan los ingredientes (tipo, cantidad y calidad) de sus preparaciones, lo que permite así un mayor control y confianza. Mientras que, por el contrario, cuando los alimentos son preparados fuera de casa, existe la duda en cuanto al tipo, calidad o cantidad de los ingredientes empleados (en el caso de los restaurantes) y para los productos elaborados industrialmente se añade la sospecha de la inclusión de aditivos "químicos" o conservantes que podrían tener un efecto negativo para la salud.

[Comida] ¿Sana?, pues no comprar comida hecha que te viene en recipiente como los macarrones con la salsa, porque no sabes muy bien lo que lleva, si lleva mucha grasa, o si lleva... por ejemplo ahora que hago bizcochos y galletas, pues claro las galletas compradas ja saber qué llevan! ¿no? [se ríe] [...] por ejemplo, haces un bizcocho en casa y al cabo de unos días ya está duro y en cambio los [bizcochos] del súper [se ríe] se pueden caducar y aún están blandos [se ríe] y pienso: "¿qué le deben de meter para que aquello [se conserven]? Aditivos y, no sé, conservantes, colorantes. No inspira mucha confianza para una alimentación sana [Julia, 24 años].

Si bien la idea de control sobre cantidades y tipo de grasa fue expresada desde el eje argumental 1 , su influjo en las PCC de las participantes evidencia cómo y dónde son convertidos los alimentos en comida.

En las PCC, las informantes refirieron poner en práctica distintas estrategias cuando la salud es un mediador en sus elecciones alimentarias: eliminación (evitar o excluir alimentos o platos específicos), limitación (restricción de alimentos, ingredientes o platos en cantidad o frecuencia de consumo), sustitución (reemplazo de ingredientes), adición (inclusión de alimentos 0 ingredientes) y modificación (cambios en recetas en relación con los procesos de cocción, conjunto de adiciones, cambios o sustituciones de ingredientes o procesos de manipulación).

Aunque el poco o nulo empleo de grasa fue asociado con una característica de la alimentación saludable, al profundizar en el discurso lipofóbico de las informantes, se identificó que esta relación es compleja y contradictoria. Además de la cantidad de grasa, se añade a la apreciación de lo "saludable" la fuente alimentaria de esta (el aceite de oliva mejor que otros), el tipo (consideradas como buenas o malas para tu salud) e incluso condiciones particulares durante su empleo en la elaboración de las comidas, como freír los alimentos con la cantidad "suficiente" de aceite y a la temperatura "adecuada"; estas forman parte de las estrategias de modificación que algunas informantes revelaron para obtener fritos "más saludables".

[...] pero también es cierto que el frito no es tan, tan malo como lo pintamos. $O$ sea, dicen que el aceite tiene que tener una temperatura muy concreta para 
que no haga daño, para que no se queme, por un lado, si es demasiado caliente, o que sí es demasiado frío, digamos, si no llega a esta temperatura concreta, los alimentos que vamos a freír se impregnan del aceite, entonces son más grasos, más pesados etcétera. Entonces, si conseguimos alcanzar esa temperatura concreta, pues el punto concreto en que el aceite en realidad no es no tóxico para el cuerpo [Alicia, 43 años].

Origen y producción de alimentos. El origen geográfico de los alimentos, su mayor proximidad, es un criterio de salud..$^{12}$ Así, los productos 0 alimentos españoles, mediterráneos, catalanes 0 de "kilómetro 0" inspiran mayor confianza, y se les atribuye mayor inocuidad que a aquellos que provienen o se producen en lugares distantes.

Los métodos intensivos de producción (en agricultura, ganadería y piscicultura) afectan la apreciación de lo "saludable" del alimento, debido al uso y a la posible incorporación (y permanencia) de sustancias (pesticidas, hormonas, fármacos, etc.) consideradas indeseables en los alimentos, pues su consumo entraña la posibilidad de repercusiones indeseables en la salud.

Lo ecológico [mejor] por salud, por sabor, por todo, el gusto sobretodo y por salud y por los aspectos [negativos] que te pueden dar los restos de productos y fungicidas usados en la agricultura [Pilar, 51 años].

Por el contrario, los métodos relacionados con la agricultura biológica ${ }^{13}$ se asociaron con alimentos "saludables", debido a que se perciben como carentes de aditivos, residuos o sustancias nocivas, a la vez que se les atribuyen características

12 Además, la cercanía, geográfica, con los productores se asocia con características deseables en los alimentos, como la "frescura" 0 el "sabor", que se relacionan con condiciones o procesos de maduración y con un "mejor" sabor de los alimentos.

13 Ecológica u orgánica si refiere a las formas de producir alimentos que evitan el uso de productos sintéticos, como pesticidas, herbicidas y fertilizantes artificiales. organolépticas $^{14}$ y nutricionales superiores, en contraste con aquellos alimentos de producción intensiva.

Aunque, en términos generales, lo elaborado fuera de casa (ya sea por la industria alimentaria o restaurantera) no sea percibido como saludable, la proximidad de los productos empleados puede matizar esta apreciación en algunas informantes, ya que los productos o alimentos regionales o nacionales se asocian, como hemos visto, con una mayor confianza y como "más saludables".

Por ejemplo, en el Bo [nombre de la cadena de distribución], tienen bastantes variedades de comida que yo considero que es bastante sana entre comillas $y$, bueno, creo que se cocina con alimentos que están hechos aquí, entonces me da más seguridad de alguna forma que estén [hechos] aquí a que vengan de lejos ¿sabes? Hay un pollo por ejemplo que está ya cocinado, viene con una salsa, hay uno de habitas con butifarra que es un plato también tipo de aquí, hay lasaña ¿qué más?, ¿qué otra cosa suelo comprar? ¡Ah! Hay un pescado, como filetes de lomo con verduras que están ya como cocinadas y eso es simplemente pinchar el taper y meterlo al microondas y me da la sensación que [...] el hecho de que sea con productos de aquí me da como más fiabilidad que estén hechos aquí ¿ sabes? Como más sano [Juana, 25 años].

\section{La salud y otras consideraciones en las prácti- cas culinarias cotidianas}

Las PCC de las informantes son influenciadas por las trayectorias y transiciones alimentarias (emancipación, cohabitar en pareja, embarazo, procreación) (19), procesos de racionalización de los recursos disponibles (principalmente dinero y tiempo) y los valores que integran el SPDA.

14 El color y el sabor fueron las más referidas y los vegetales, los más nombrados. 
Entre los diversos valores y factores que las mujeres consideran en sus decisiones alimentarias cuando focalizan la importancia de la salud, tres valores en especial aparecen con más frecuencia en la conciliación, negociación y jerarquización por encima de la salud: el primero se refiere a los gustos y preferencias alimentarias (GPA) personales y de los otros para quienes se cocina (generalmente pareja o hijos); el segundo es el costo (relativo al dinero del que se dispone y su relación con el precio de los insumos); y el tercero trata de la conveniencia, es decir, de la "practicidad" o inmediatez en relación con el empleo mínimo de tiempo en la elaboración o consumo de las comidas.

Los procesos de jerarquización permiten mitigar la tensión y conflicto de las diferentes consideraciones que se toman en cuenta en las PCC de las informantes. Esto permite entender los distanciamientos entre los discursos y las prácticas en el marco de lo que es o no percibido como "saludable".

El valor de los GPA fue uno de los más apreciados y menos sujetos a negociación. Esto explica el porqué, en algunas elecciones de alimentos, aunque estos son considerados "no saludables", son consumidos. Al respecto, se identificaron varios casos en las PCC en los que las informantes presentan el consumo de leche como ejemplo. En efecto, aunque percibida como "no saludable" (por considerarla no necesaria en la etapa de la adultez y por las sustancias que, se presume, se integran a través de los métodos de producción masivos de alimentos), Mercedes bebe leche para satisfacer sus GPA.

[...] me gustaría quitarme el hábito de la leche de vaca pero me resulta difícil porque me gusta, sería lo ideal [porque] la leche de vaca llega un momento que no la necesitamos porque estamos bebiendo leche de un animal que no es de nuestra especie, ya hemos bebido leche de nuestra madre [...] es producción masiva con lo cual es un animal que le dan hormonas para que produzca [...] yo reconozco que a mí me gusta la leche, entonces compro leche fresca y estoy a la mar de contenta [risas], pero, claro, reconozco que la leche de vaca tendría que [dejar] ...he llegado a esta conclusión para cuando sea mayor quiero quitarme la leche [se ríe] aunque me guste, pero me resulta difícil, me gusta la leche de avena, pero ¿ ¡café con leche de avena!?, jno!, no es lo mismo al café con leche [de vaca] [Mercedes, 45 años].

Por otro lado, los recursos disponibles, sobre todo en tiempo y dinero, y que se manifiestan en los valores de costo y conveniencia, influyen, también, con mayor importancia que la salud en las PCC.

En el caso del costo, algunas informantes reconocieron la constricción del costo en algunos alimentos considerados como "saludables" y por ello limitan su consumo. Este fue el caso especialmente detectado en los alimentos bio.

A mí me gusta comprar ecológico, pero no siempre puedo por economía. Me gusta [...] el sabor, sobre todo las verduras, sobre todo [...] por salud, por sabor, por todo, pero no siempre puedo [Pilar, 51 años].

El tiempo es un mediador importante en las PCC. La apreciación de falta de este recurso se agudiza por el desempeño de las actividades de los diversos roles que llevan a cabo las informantes. En este sentido, la conciliación de la gestión del hogar y la familia con el desempeño de un trabajo remunerado fueron los factores que más influyeron en la apreciación de falta de tiempo y que, a su vez, producen sensaciones de agobio, de "ir siempre de prisa". Estas condiciones repercuten negativamente en la apreciación de disposición de energía física (que incrementa una noción de agotamiento o cansancio) y que también repercute en la falta de motivación personal para realizar las actividades culinarias. 
Por tanto, cuando no se dispone de suficiente tiempo, energía o motivación para el desempeño de las tareas culinarias, el valor de la conveniencia influye en las PCC de las informantes. Así, aunque los alimentos precocinados o "listos para comer" no fueron percibidos "saludables", entre la mayoría de las informantes, estos forman parte del repertorio cotidiano de elecciones alimentarias de las mujeres, como muestra el testimonio de Dulce, al señalar reiteradamente su apreciación de que un alimento preparado en casa es más "saludable", pero consumía alimentos precocinados.

Los [canelones los] tienes que cuidar que no se enganchen [mientras se cuece la pasta], eso me da un coraje, yo muchas veces los compro hechos. Aunque es un poco más caro, pero ya hay esas cosas que ya están hechas y bueno, te ahorras tiempo [Dulce, 69 años].

El ahorro de tiempo, bien valorado en los productos listos para consumir, también fue referido tanto en la planificación como en las tareas de pre y poslimpieza.

\section{DISCUSIÓN}

Lo que se percibe como "saludable" evidencia la reproducción de discursos que ponen de manifiesto la nutricionalización de la alimentación (2). Los discursos de las informantes relativos a tipos de alimentos, nutrientes, cantidades y frecuencias coinciden con algunas de las normas dietéticas (22) 0 de las pautas basadas en conocimientos científicos y difundidas por actores del ámbito sanitario (9). Reflejan, asimismo, procesos de asimilación y resignificación (matices, tensiones y contradicciones), mediados por las trayectorias y transiciones alimentarias (19) e influenciados por la cacofonía alimentaria, información alimentaria con frecuencia contradictoria, difundida por diversos actores a través de distintos canales de comunicación (23), que varía con el tiempo (8) y a la que han estado expuestas las mujeres entrevistadas durante su itinerario vital.

A partir de procesos de resignificación, lo que se percibe como "saludable" trasciende la óptica estrictamente nutricional e incluye otros matices, tensiones y contradicciones. Un ejemplo son las contradicciones identificadas en torno a las narrativas lipofóbicas de las participantes. En efecto, los discursos lipofóbicos y la diferenciación de los tipos de grasas relatados por las informantes evidencian la reproducción de discursos dietéticos y la medicalización alimentaria (1); pero, también, las resignificaciones que se contraponen de acuerdo con parámetros específicos en las PCC, al considerar que los alimentos fritos percibidos puedan ser "más saludables".

También se han identificado otras condiciones ambivalentes sobre lo relativo a la dicotomía "saludable-no saludable" de los alimentos (en particular el consumo de la leche), los lugares de elaboración de las comidas, el origen y los métodos de producción de alimentos.

Para entender la discrepancia entre lo que es percibido como "saludable" y la realidad de las PCC, se presentan dos acercamientos: el primero son las nociones de asimilación y resignificación y el segundo, las ideas constantes de necesidad de identificación, control y confianza que emergieron reiteradamente en los discursos de las informantes.

Los procesos de asimilación y resignificación se entrelazan y el primero permite la integración del segundo. La asimilación de los discursos nutricionales (en condiciones de cacofonía alimentaria) se realiza progresiva y discontinuamente a lo largo de la vida; la información obtenida es de carácter fragmentario (8). Cuanto más próximos sean los fragmentos de información y las categorías cognitivas preestablecidas o con los GPA de las 
personas, se incrementan las posibilidades de asimilación de la información, pues esta se engrana con las categorías mentales ya interiorizadas. Dicho engranaje da lugar a procesos de resignificación. Los procesos de resignificación aquí presentados permiten explicar por qué las informantes perciben que "saludable" incluye, también, lugares de elaboración de las comidas, origen y métodos de producción de alimentos, variedad, equilibrio y dieta mediterránea. Según Martín Criado (8), "lo natural es lo de aquí - del pueblo, de la región — frente a lo de fuera. También es lo tradicional, lo conocido de toda la vida, frente a los alimentos y preparaciones nuevas" (p. 522). De acuerdo con el autor, entre más generales sean los preceptos (ej. alimentación equilibrada, dieta mediterránea), más probables las reinterpretaciones y distorsiones.

Se detectaron tres imperantes en la percepción de lo "saludable": la identificación, el control y la confianza que influyen en cuan "saludables" son percibidos alimentos, nutrientes, modos de preparación o producción de alimentos, equipo y menaje de cocina y procedencia de los alimentos. Esto puede explicarse a partir de los principios de incorporación y de mediación culinaria propuestos (23) y el riesgo alimentario (24), que se refieren a la incertidumbre que entraña la integración de sustancias indeseables en el cuerpo por la falta de control o de identificación.

De acuerdo con Fischler (23), al comer, el comiente incorpora el alimento. Tanto en el "plano real como en el plano imaginario, incorpora todo o parte de sus propiedades. Así, la comida constituye al humano, en tanto a la preservación de la vida, la salud, la identidad y la alteridad" (pp. 65-6); todas razones determinantes que explican la necesidad de identificación (en el sentido propio y figurado) detectada en los discursos de las informantes.
La identificación de lo que se come, en el caso de la cocina doméstica, se lleva a cabo por la mediación culinaria (23). Las informantes tuvieron la oportunidad de identificar tipo, cantidad y calidad de los ingredientes empleados para la elaboración de sus platos y esto les brindó la sensación de control y confianza, pues reconocieron e identificaron fácilmente el alimento que consumieron.

Por el contrario, este sentido de confianza, que puede generarse en la cocina doméstica por la mediación culinaria, puede transgredirse en el caso de desconocimiento por parte de los consumidores respecto de los modos de fabricación de los alimentos y de las verdaderas materias primas utilizadas en los alimentos que son producidos 0 procesados industrialmente. Este desconocimiento alimenta la percepción de riesgo alimentario (24), al caber la sospecha de que puedan haber sido incorporadas sustancias percibidas como tóxicas o peligrosas (aditivos, pesticidas, hormonas, etc.) al cuerpo. Considerado así, no es de extrañar que la apreciación de desconfianza, de "no saludable" o de riesgoso para la salud de los alimentos que son producidos y procesados industrialmente, además de lo encontrado en este estudio, también haya sido advertida por otros investigadores $(25,26)$.

Sobre la tensión entre la constante búsqueda de equilibrio y variedad, destacada por algunas informantes, se considera que la paradoja del omnívoro (23) podría ayudar a entender la tensión expresada por algunas mujeres. De acuerdo con Fischler (23), por un lado, el omnívoro disfruta de autonomía y libertad en la elección de alimentos; pero, por otro, necesita de un mínimo de variedad para disponer de todos los nutrientes biológicamente requeridos por los humanos. Así, la libertad de elección se asocia con la dependencia y una coacción: la variedad. Condición que crea tensión entre los comportamientos relativos 
a la diversificación, innovación y exploración (neofilia) en la búsqueda de la variedad y la prudencia, desconfianza y conservadurismos alimentarios (neofobia), ya que todo alimento desconocido supone un peligro potencial. La tensión entre los dos polos (neofobia y neofilia) es fuente de angustia y ansiedad en el comiente.

Bajo este marco, pareciera que al menos en el caso de algunas de las informantes, cocineras del siglo XXI y que desempeñan diversos roles, la sensación de ansiedad y angustia relacionada con la paradoja del omnívoro se experimenta por duplicado: uno por la incertidumbre y desconfianza derivadas de la no identificación de los alimentos más o menos procesados $y$, dos, por buscar la satisfacción y la variedad en la planificación y preparación de las comidas. Ambas sensaciones en un marco alimentario que, paradójicamente, también es percibido como variado.

El influjo de la salud en las PCC y en las estrategias de limitación, modificación, etc., identificadas en el presente estudio, también han sido señaladas por otros autores (19). Por ejemplo, Falk et al. (27) destacan que la priorización de valores que las personas realizan para tomar una decisión es en sí misma una estrategia en la cual suelen "eliminar la consideración de uno o más valores” (p. 197).

En concordancia con otros autores, el presente estudio pone de manifiesto la falta de correspondencia (o el alejamiento en grados variables) de las PCC con las pautas nutricionales establecidas por expertos, y ello se debe a que la salud, como otro valor más de los que integran SPDA es jerarquizado con relación a los GPA $(3,8,28)$, el costo $(2,29-31)$ y la conveniencia $(2,4,32,33)$ que se articulan con otros factores materiales y simbóli$\cos (3,34,35)$ bajo influjos socioculturales para dar lugar a una decisión alimentaria.
En conclusión, las percepciones de lo que se considera "saludable" son complejas y diversas. Complejas porque se configuran a partir de normas sociales y dietéticas que están sujetas a modificaciones durante las trayectorias y transiciones del itinerario vital y que reflejan las experiencias sociales y culturales, a la vez que ponen de manifiesto los individualismos alimentarios y los procesos de apropiación y resignificación de los distintos discursos sobre alimentación y cacofonía alimentaria reinante.

Son diversas debido a que admiten matices, tensiones y contradicciones que se relacionan con la ingestión -o la privación del consumo- de determinados alimentos 0 grupos de alimentos, nutrientes concretos, patrones alimentarios especificos, frecuencias o cantidades de consumo, formas o lugares de preparar o producir los alimentos.

Si bien la salud fue referida como elemento importante de las PCC de la muestra estudiada, cabe considerarla más como un referente moral en el plano discursivo para orientar tanto el comportamiento alimentario propio como el de los demás. Sin embargo, al estudiar las PCC y la complejidad que le es inherente, hay que concluir que la salud no es ni el único valor ni necesariamente el más apreciado en la toma de decisiones relativas a las PCC de las mujeres que participaron en el estudio.

Las resignificaciones sobre to que se considera como "saludable" facilitan a las informantes integrar diversas estrategias y legitimar los discursos sobre salud en sus PCC. Condición que explica, desde las perspectivas de las informantes, que, en efecto, el valor de la salud sea considerado en sus elecciones alimentarias. Aunque ello no necesariamente se corresponda con las normas dietéticas.

Reconocer la complejidad del hecho alimentario y comprender cómo la gente asimila, interpreta y 
resignifica su alimentación es importante ya que ello podría proporcionar elementos a los profesionales de la salud para brindar educación nutricional al ayudar a las personas a identificar y corregir posibles distorsiones 0 malas interpretaciones entre las pautas dietéticas recomendadas y cómo son llevadas a la práctica cotidiana; también, para, desde la propia subjetividad de las personas, emitir recomendaciones dietéticas más acordes con la vida cotidiana de las personas, en la medida de lo posible, más cercanas a sus referentes y, así, incrementar la posibilidad de orientar con mayor éxito a las personas con el fin de que, si así lo deciden, mejoren sus elecciones alimentarias, desde una perspectiva nutricional.
Para futuras investigaciones, es conveniente explorar cómo se llevan a cabo los procesos de asimilación y resignificación de la salud y la alimentación en el ámbito doméstico en otros grupos (incluir hombres, con otros referentes culturales

\section{DECLARACIÓN DE CONFLICTO DE INTERES}

Los autores manifestaron no tener conflicto de intereses.

\section{AGRADECIMIENTOS}

Trabajo realizado gracias al financiamiento otorgado por la Secretaria de Educación a través Programa de Mejoramiento del Profesorado (PROMEP), ahora PRODEP.

\section{-Referencias}

1. Conrad P. Medicalization and social control. AnnuRevSociol. 1992;18(1):209-32. https://doi.org/10.1146/annurev.so.18.080192.001233

2. Gaspar MC, Juzwiak C, Muñoz A, Larrea-Killinger C. Las relaciones entre salud y alimentación. Una lectura antropológica. En: Observatorio de la Alimentación ODELA. Polisemias de la Alimentación Salud, desperdicio, hambre y patrimonio. Barcelona: Ediciones de la Universitat de Barcelona; 2018, pp. 53-75.

3. Contreras J, Gracia M. Comemos como vivimos. Alimentación, salud y estilos de vida. Barcelona: Alimentaria Exhibitions; 2006, $129 \mathrm{pp}$.

4. Lappalainen R, Kearney J, Gibney M. A pan EU survey of consumer attitudes to food, nutrition and health: An overview. Food Qual Prefer. 1998;9(6):467-78. https://doi.org/10.1016/S0950-3293(98)00018-4

5. Lennernäs M, Fjellström C, Becker W, Giachetti I, Schimitt A, Remaut de Winter A, et al. Influences on food choice perceived to be important by nationally-representative samples of adults in the European Union. Eur J Clin Nutr. 1997;51(Suppl 2):S8-15. https://pubmed.ncbi.nlm.nih.gov/9222718/

6. Wardle J, Haase AM, Steptoe A, Nillapun M, Jonwutiwes K, Bellisie F. Gender differences in food choice: The contribution of health beliefs and dieting. Ann Behav Med. 2004;27(2):107-16. https://doi.org/10.1207/s15324796abm2702_5

7. Kandrack MA, Grant KR, Segall A. Gender differences in health related behaviour: Some unanswered questions. SocSciMed. 1991;32(5):579-90. https://doi.org/10.1016/0277-9536(91)90293-L

8. Criado M. El conocimiento nutricional apenas altera las prácticas de alimentación: el caso de las madres de clases populares en Andalucía. RevEsp Salud Publica. 2007;81(5):519-28. https://doi.org/10.1590/S1135-57272007000500008

9. Contreras-Hernádez J, Gracia-Arnaiz M. VI. Preferencias y consumos alimentarios: entre el placer, la conveniencia y la salud. En Alimentación, consumo y salud. Barcelona: Fundación "la Caixa"; 2008. pp. 153-91. 
10. Contreras J, Gracia M. La alimentación y sus circunstancias: placer, conveniencia y salud. Primera. Barcelona: Alimentaria; 2004, $231 \mathrm{pp}$.

11. López-Torres L, Navia B, Ortega R. Percepción sobre la calidad de la dieta en un colectivo de adultos. Comparación con la calidad real. Nutrclíndiethosp. 2017;37(2):75-82. https://doi.org/10.12873/372lopez

12. Varela-Moreiras G, Ávila JM, Cuadrado C, del Pozo S, Ruiz E, Moreiras O. Evaluation of food consumption and dietary patterns in Spain by the Food Consumption Survey: Updated information. Eur J Clin Nutr. 2010;64:S37-43. https://doi.org/10.1038/ejcn.2010.208

13. Beagan B, Chapman G, D'Sylva A, Bassett B. 'It's Just Easier for Me to Do It': Rationalizing the Family Division of Foodwork. Sociology. 2008;42(4):653-71. https://doi.org/10.1177/0038038508091621

14. Martínez M. Métodos fenomenológicos. En: Ciencia y Arte en la Metodología Cualitativa. México: Trillas; 2015, pp. 137-79.

15. Giard L. Hacer de comer. En: Certeau M, Giard L, Mayol P (Eds.). La invención de lo cotidiano 2. Habitar, cocinar. México, D.F.: Universidad lberoamericana; 1999, $271 \mathrm{pp}$.

16. Short F. Kitchen secrets: The meaning of cooking in everyday life. New York: Berg; 2006, 224 pp.

17. Harris $\mathrm{J}$, Gleason $\mathrm{P}$, Sheean $\mathrm{P}$, Boushey $\mathrm{C}$, Beto J, Bruemmer B. An introduction to qualitative research for food and nutrition professionals. J Am Diet Assoc. 2009;109(1):80-90. https://doi.org/10.1016/j.jada.2008.10.018

18. Glaser B, Strauss A. The discovery of grounded theory strategies for qualitative research. United States of America: Aldine Transaction; 1999, $282 \mathrm{pp}$.

19. Sobal J, Bisogni C. Constructing food choice decisions. Ann Behav Med. 2009;38(SUPPL. 1)S37-46. https://doi.org/10.1007/s12160-009-9124-5

20. CIOMS, OMS. Pautas éticas internacionales para la investigación biomédica en seres humanos. Santiago, Chile: 2002.

21. Agar L. La ética de la investigación en ciencias sociales en el contexto de la globalización: de la investigación cuantitativa a la cualitativa. Acta Bioeth. 2004;10(1):65-8. https://doi.org/10.4067/S1726-569X2004000100008

22. Ortega RM, Requejo AM, Andrés P, Redondo MR, López-Sobaler AM, Quintas E NB. El Rombo de la Alimentación. Guía útil en la planificación de dietas ajustadas a las pautas recomendadas. Nutr Clínica. 2006;XXVI/47(2):31-9.

23. Fischler C. El (h)omnívoro. El gusto, la cocina y el cuerpo. Barcelona: Anagrama; 1995, 422 pp.

24. Larrea-Killinger C, Muñoz A, Mascaró J. Cuerpos tóxicos: la percepción del riesgo de la contaminación interna por compuestos químicos en España. SaludColect. 2017;13(2):225. https://doi.org/10.18294/sc.2017.1161

25. Bisogni C, Jastran M, Seligson M, Thompson A. How people interpret healthy eating: Contributions of qualitative research. J Nutr Educ Behav. 2012;44(4):282-301. https://doi.org/10.1016/j.jneb.2011.11.009

26. Neuman N, Persson C, Mattsson $Y$, Fjellström C. Swedish students' interpretations of food symbols and their perceptions of healthy eating. An exploratory study. Appetite. 2014;82:29-35. https://doi.org/10.1016/j.appet.2014.07.003

27. Falk L, Sobal J, Bisogni C, Connors M, Devine C. Managing healthy eating: Definitions, classifications, and strategies. Heal Educ Behav. 2001;28(4):425-39. https://doi.org/10.1177/109019810102800405

28. Slater J, Sevenhuysen G, Edginton B, O'neil J. "Trying to make it all come together": Structuration and employed mothers' experience of family food provisioning in Canada. Health Promot Int. 2012;27(3):405-15. https://doi.org/10.1093/heapro/dar037

29. Engler-Stringer R. The domestic foodscapes of young low-income women in Montreal: Cooking practices in the context of an increasingly processed food supply. HealEducBehav. 2010;37(2):211-26. https://doi.org/10.1177/1090198109339453 


\section{Percepciones sobre la alimentación saludable}

30. Hernández M. A la altura de lo cotidiano: algunos sentidos del cocinar y comer en casa. Fundam Humanidades. 2008;II(18):71-89. https://www.redalyc.org/articulo.oa?id=18411970004

31. Reicks M, Trofholz A, Stang J, Laska M. Impact of cooking and home food preparation interventions among adults: outcomes and implications for future programs. J Nutr Educ Behav. 2014;46(4):259-76. https://doi.org/10.1016/j.jneb.2014.02.001

32. Daniels S, Glorieux I. Convenience, food and family lives. A socio-typological study of household food expenditures in 21st-century Belgium. Appetite. 2015;94:54-61. https://doi.org/10.1016/j.appet.2015.04.074

33. Gracia M. Maneras de comer hoy. Comprender la modernidad alimentaria desde y más allá de las normas. Rev Int Sociol. 2005;63(40):159-82. https://doi.org/10.3989/ris.2005.140.193

34. Antin T, Hunt G. Food choice as a multidimensional experience. A qualitative study with young African American women. Appetite. 2012;58(3):856-63. https://doi.org/10.1016/j.appet.2012.01.021

35. Gracia M. De modernidades y alimentación: comer hoy en España. Horizontes Antropológicos. 2010;16(33):177-96. https://doi.org/10.1590/S0104-71832010000100010

Anexo. Guion

\begin{tabular}{|c|c|}
\hline Tema & Preguntas \\
\hline Percepciones de la cocina & $\begin{array}{l}\text { Hay encuestas que dicen que el } 51 \text { \% de las españolas consideran que no se sigue una alimentación } \\
\text { más acorde a su salud porque hace falta educación nutricional a ¿que faltas se pueden referir? }\end{array}$ \\
\hline Percepciones Saludable & $\begin{array}{l}\text { El } 49 \text { \% dice que son otros los motivos y no la falta de educación nutricional ¿cuáles creen que son } \\
\text { estos motivos? } \\
\text { ¿Qué consideras que es una alimentación saludable? ¿Puedes describirme cuáles son las caracte- } \\
\text { rísticas que tiene una alimentación saludable? ¿Podrías darme ejemplos de una alimentación (hábi- } \\
\text { tos, comidas, platos, recetas) saludable? }\end{array}$ \\
\hline Planeación & $\begin{array}{l}\text { ¿Con que frecuencia cocinan? — todos los días y veces por día- } \\
\text { ¿Cómo saben —-planean- que hacer de comer? En ocasiones descrita como una actividad que } \\
\text { cansa } \\
\text { ¿Qué diferencias hay en los menús que preparan los días de la semana y el fin de semana? }\end{array}$ \\
\hline Compra & $\begin{array}{l}\text { ¿Cómo deciden que hay que comprar? } \\
\text { ¿Qué alimentos procuran tener siempre en la alacena? Para una comida rápida no hay tiempo }\end{array}$ \\
\hline Preparación & $\begin{array}{l}\text { ¿Cuáles técnicas culinarias-preparaciones de alimentos consideran que son las más saludables? Y } \\
\text { ¿por qué? Tips para una comida rápida, fácil y saludable. } \\
\text { Hay encuestas en las que las mujeres opinan que } 2 \text { de las } 3 \text { principales razones para explicar el } \\
\text { empeoramiento de nuestros hábitos alimenticios son: } \\
\text { 1. Buscamos la comodidad y los platos precocinados porque son fáciles de preparar, aunque no } \\
\text { siempre sean saludables. } 2 \text {. Podríamos comer mejor que nunca, pero cada vez tenemos más obli- } \\
\text { gaciones a las que responder y nos queda poco tiempo y ganas para cocinar. ¿Ustedes que opinan? }\end{array}$ \\
\hline Equipo & ¿De qué electrodomésticos —equipo— disponen en sus casas y por qué? \\
\hline $\begin{array}{l}\text { Participación de los hombres y } \\
\text { otros miembros del grupo do- } \\
\text { méstico }\end{array}$ & $\begin{array}{l}\text { ¿De qué manera sus parejas participan en la cocina? / ¿Saben cómo aprendieron ellos a cocinar? } \\
\text { ¿De qué manera participan los otros miembros de la familia en la cocina? }\end{array}$ \\
\hline
\end{tabular}

